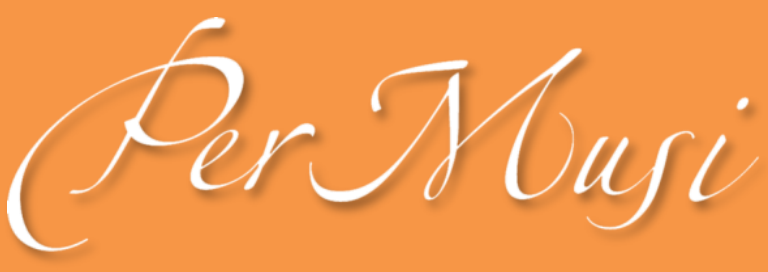

eISSN 2317-6377

\title{
Análisis del bienestar psicológico en la práctica coral inclusiva
}

\author{
M. Belén López-Casanova \\ https://orcid.org/0000-0001-9101-6478 \\ Universidad de Zaragoza, Departamento de \\ Expresión Musical, Plástica y Corporal \\ belocasa@unizar.es \\ Icíar Nadal-García \\ https://orcid.org/0000-0003-1325-2373 \\ Universidad de Zaragoza, Departamento de \\ Expresión Musical, Plástica y Corporal \\ iciarnad@unizar.es
}

\author{
Natalia Larraz-Rábanos \\ https://orcid.org/0000-0001-7091-2855 \\ Universidad de Zaragoza, Departamento de \\ Psicología y Sociología \\ nlarraz@unizar.es \\ Borja Juan-Morera \\ https://orcid.org/0000-0002-5943-5012 \\ Universidad de Zaragoza, Departamento de \\ Expresión Musical, Plástica y Corporal \\ bjuan@unizar.es \\ SCIENTIFIC ARTICLE \\ Submitted date: 13 jul 2021 \\ Final approval date: 27 sep 2021
}

Resumen: La práctica coral aporta considerables beneficios en el ámbito psicológico, afectivo y social, que favorecen la inclusión y cohesión social. Se presenta un estudio realizado en un coro inclusivo universitario. El objetivo es realizar un análisis descriptivo de la situación actual del coro para adaptar y diseñar actuaciones que contribuyan a la mejora del bienestar psicológico. La muestra está compuesta por 73 participantes. El método empleado es de investigación cuantitativa ex post facto, con carácter retrospectivo y comparativo. Los resultados revelan que existen diferencias en el bienestar psicológico con respecto a la diversidad de género y en aquellos individuos que perciben malestar psicológico. Las personas con diversidad funcional no son aquellas que parecen tener menor bienestar psicológico con respecto a aquellas sin diversidad, aunque sus niveles de bienestar psicológico sean menores. Este aspecto invita a concluir que las actividades planteadas en el coro podrían potenciar mayores niveles de bienestar psicológico.

Palabras clave: Música; Inclusión; Canto coral; Diversidad; Bienestar psicológico.

\section{TITLE: ANALYSIS OF PSYCHOLOGICAL WELL-BEING IN INCLUSIVE CHORAL PRACTICE}

Abstract: Choral practice provides considerable benefits in the psychological, affective and social spheres, which favour inclusion and social cohesion. A study carried out with the participants of an inclusive choir. The objective is to carry out a descriptive analysis of the current situation of this choir to adapt and design actions that contribute to the improvement of psychological well-being. The sample is made up of 73 participants. The method used is ex post facto quantitative research, retrospective and comparative. The results reveal that there are differences in psychological well-being with respect to gender diversity and in those individuals who perceive psychological distress. People with functional diversity are not those who seem to have lower psychological well-being than those without functional diversity, although their levels of psychological well-being are lower. This aspect invites us to conclude that the activities proposed in the choir could enhance higher levels of psychological well-being.

Keywords: Music; Inclusion; Coral singing; Diversity; Psychological well-being. 


\section{Análisis del bienestar psicológico en la práctica coral inclusiva}

M. Belén López-Casanova, Universidad de Zaragoza, belocasa@unizar.es

Icíar Nadal-García, Universidad de Zaragoza, iciarnad@unizar.es

Natalia Larraz-Rábanos, Universidad de Zaragoza, nlarraz@unizar.es

Borja Juan-Morera, Universidad de Zaragoza, bjuan@unizar.es

\section{Introducción}

Los beneficios del canto colectivo son múltiples para las personas que lo practican, así lo corroboran los diferentes estudios que se han llevado a cabo en los últimos años. La literatura científica (Broh 2002; Fernández Herranz 2013; Hallam 2010; Pieridou 2018; Whitwell 1977), constata las mejoras en el ámbito psicológico y social que la música, en general, y el canto colectivo, en particular, generan en los individuos. Estas experiencias favorecen la autoestima, la motivación, la confianza en sí mismo y en los demás, la autoeficacia, las habilidades sociales, el bienestar y los sentimientos positivos como la alegría, el amor, el sentido de realización y de pertenencia, el aprecio y el respeto, etc.

El canto coral es una actividad social y creativa, con valor intrínseco y recompensas para quienes la practican y también para el público que la disfruta (Clift et al. 2010a), además se debe llevar a cabo de manera que sea accesible para todos los ciudadanos y favorecer la inclusión. En este sentido, el estudio llevado a cabo por Juan, Nadal y López (2020), sugiere que a través de la música coral, a su vez, interpretada-traducida a la Lengua de signos, se llega a un mayor número de público y la música se hace más accesible para las personas con y sin diversidad funcional.

La música estimula las conexiones cerebrales que activan las emociones, y colabora en el proceso del desarrollo psíquico y afectivo que facilita el equilibrio adecuado para lograr la mejora del bienestar (Lacárcel 2003).

En este artículo se muestran los resultados recogidos a través de una escala de bienestar psicológico en un coro inclusivo en el que sus participantes tienen diversos perfiles. Se trata de realizar un análisis descriptivo de la situación actual del coro para poder adaptar y diseñar los diferentes tipos de actuaciones que contribuyan a la mejora del bienestar psicológico como compromiso de responsabilidad social. Esta necesidad se hace imprescindible en la situación actual de pandemia provocada por la Covid-19. 


\section{Revisión de la literatura}

\subsection{Bienestar psicológico y bienestar subjetivo}

Blanco y Díaz (2006), en base a las investigaciones realizadas por Díaz y Sánchez (2002) y Diener et al. (1994), sostienen que existen dos amplias tradiciones: la hedónica que se ocupa del bienestar subjetivo y la eudaimónica que representa al bienestar psicológico. Según Díaz et al. (2006), así como Blanco y Díaz (2006), el bienestar subjetivo hace referencia al estudio de los afectos y la satisfacción con la vida, mientras que el bienestar psicológico tiene como base el desarrollo personal y el fomento del estilo y la manera de afrontar retos en el esfuerzo y el afán por lograr nuestras metas vitales. Blanco y Díaz (2006) indican que ambos constructos están relacionados, pero empíricamente diferenciados, poniendo énfasis en los rasgos y estilos de personalidad en el bienestar subjetivo y en variables sociodemográficas como el sexo, la edad, el estado civil, el estatus socioeconómico y el nivel educativo en el bienestar psicológico.

Se podría entender que el bienestar psicológico es un constructo multidimensional compuesto por seis dimensiones: autoaceptación, relaciones positivas con otras personas, autonomía, dominio del entorno, propósito en la vida y crecimiento personal (Blanco y Díaz 2006; Ryff 1989a; 1989b). La autoaceptación se entiende como un criterio central del bienestar, implica intentar sentirse bien con uno mismo siendo consciente incluso de las propias limitaciones, tener actitudes positivas hacia uno mismo. Las relaciones positivas con otras personas implican mantener relaciones sociales estables y tener amigos en los que poder confiar, así como la capacidad de amar. La autonomía implica poder sostener la propia individualidad en diferentes contextos sociales, asentarse en las propias convicciones (autodeterminación) y mantener la independencia y autoridad personal. El dominio del entorno, es la habilidad personal para elegir o crear entornos favorables para satisfacer los deseos y necesidades propias. Finalmente, el propósito en la vida implica la posibilidad de marcarse metas, definir una serie de objetivos que doten a la vida de un cierto sentido.

Para Mújica et al. (2018), la percepción emocional no tiene por qué ser buena o mala, sin embargo, en el entorno educativo sí que es posible evaluarla en términos positivos o negativos. Las causas y consecuencias de esa percepción emocional afectan a otros procesos psicosociales como el bienestar, la motivación, la inclusión y la justicia social, entre otros. En este sentido, se hace necesario investigar, ya que si se promueve la inclusión de estrategias pedagógicas y experiencias musicales que ayuden a desarrollar emociones positivas, podremos mejorar el bienestar psicológico de las personas inmersas en estas actividades. A su vez, dicho bienestar psicológico va a conllevar una mejora del bienestar social de las personas implicadas en ellas, dado que el bienestar personal se vincula con bienestar social de manera indisoluble (Blanco y Díaz 2006). Desde esta perspectiva se entiende que el desarrollo de acciones educativas musicales que fomenten la mejora de los estados emocionales en colectivos sociales en riesgo de exclusión social, contribuye a la inclusión y cohesión social. La participación en actividades culturales conjuntas que impliquen respeto, convivencia e igualdad de oportunidades y que sean sensibles a la diferencia favorecen la justicia social, entendida ésta como un concepto multidimensional que incluye redistribución de bienes primarios, reconocimiento de las diferencias y la participación y representación de todas las personas a nivel social (Murillo y Hernández-Castilla 2014). 
Por consiguiente, la participación en actividades musicales grupales, como un coro inclusivo, es un medio muy apropiado para promover una práctica inclusiva de responsabilidad social.

\subsection{Bienestar psicológico y canto colectivo}

La música es una de las actividades más favorables para promover el bienestar psicológico. La investigación sobre el impacto de la participación de la música en el desarrollo psicosocial tiende a basarse en autoinformes, ya sean cuestionarios o entrevistas. Los estudios realizados por Broh (2002) y Dingle et al. (2019) muestran que los beneficios psicosociales que conlleva la experiencia musical conducen a la mejora de la autoestima y a su vez a una mayor motivación y autoeficacia. Estos sentimientos generales de confianza y autoestima que genera la participación creativa en la música también aumentan la motivación para estudiar (Whitwell 1977). En la misma línea, el compromiso con la música mejora la percepción de uno mismo, pero solo si proporciona experiencias de aprendizaje positivas que sean gratificantes (Hallam 2010). Las prácticas musicales deben ser motivadoras y aportar retos y oportunidades accesibles para todos.

Los sentimientos positivos, como alegría, amor, sentido de realización, pertenencia y sentido de control, aprecio y respeto son parte necesaria e indispensable del trabajo artístico y educativo intercultural y de la supervivencia, expresión, transformación y convivencia pacífica del ser humano en la heterogeneidad y diversidad (Pieridou Skoutella 2018). La práctica musical es una experiencia que contribuye de forma activa a la inclusión como compromiso de responsabilidad social.

La experiencia musical provoca un importante disfrute y satisfacción tanto individual como colectiva, y fomenta el trabajo en grupo (Cámara 2003; Cohen et al. 2006; Elefant et al. 2012; Lally 2009). Además, el canto colectivo favorece la confianza en sí mismo, y contribuye al desarrollo de identidad dentro del grupo. En el mismo sentido, Clift \& Hancox (2001) y Hallam (2010) muestran que la participación en grupos musicales motiva la amistad, autoconfianza y promueve las nuevas relaciones sociales. El desarrollo de un trabajo musical sistémico sobre educación audioperceptiva y canto grupal, incide positivamente en la mejora del bienestar subjetivo de sus participantes (Sarfson y Larraz 2017).

Cualquier actividad de ocio que ofrezca oportunidades de mejora, dominio de una nueva habilidad, o un sentido de logro tiene el potencial de tener un efecto beneficioso en nuestro bienestar psicológico. Al respecto, Lonslade \& Day (2020) evidenciaron que las personas que participan en un coro presentaban niveles similares de bienestar, felicidad, ansiedad, depresión y autoestima que quienes participaron en otras actividades como: tocar en una orquesta, cantar de solista, tocar un instrumento como solista, practicar deporte en grupo o deporte individual. Sin embargo, los participantes que realizaban su actividad como parte de un grupo o equipo (es decir, cantantes de coros, músicos de una banda u orquesta y equipos de deportistas) presentaban niveles más altos de conexión social que aquellos que participaron en actividades similares en solitario (es decir, cantantes solistas, músicos solistas y deportista individual).

El proceso de montaje de un repertorio vocal conlleva el trabajo y la potenciación de rutinas que pueden resultar beneficiosas para el desarrollo cognitivo y emocional de sus componentes (Gelabert 2017), entre otras, el fomento del trabajo grupal y cooperativo. Asimismo, Varvarigou (2018) afirma que cuando el aprendizaje del repertorio musical llevado a cabo en grupo se realiza de oído, promueve la cohesión grupal a través de la escucha colaborativa. La participación activa como intérprete -no profesional- o pasiva como 
oyente, en la música, promueve la cohesión de los grupos humanos, la solidaridad y, en muchos casos, la identidad cultural (Valentine \& Evans 2001).

Cohen et al. (2006), encontraron mejoras significativas tanto a nivel de salud mental como físico en las personas mayores que practican el canto colectivo. En este sentido Kuhn (2002), en su investigación, realizó pruebas con saliva a los coristas antes y después de los ensayos y sus resultados evidenciaron que participar en un coro mejora el estado inmunológico de sus componentes. Igualmente, los estudios realizados por Clift \& Hancox (2001); Cohen et al. (2006); Dingle et al. (2019); Hallam (2010); Muhip \& Gorseu (2014); Pearce et al. (2016), evidencian que cantar en un coro contribuye positivamente en la salud física y ayudan al control del estrés y autocontrol frente a la ansiedad.

También, desde el campo de la musicoterapia, Eyre (2011) estudió las relaciones entre la actividad coral y la calidad de vida, comprobando que incide en la gestión del estrés, alteraciones del humor y expresión de las emociones. La creación de música en grupo contribuye al bienestar a lo largo de la vida y, por lo tanto, puede favorecer a la cohesión comunitaria proporcionando beneficios para la sociedad en su conjunto (Hallam 2010). A este respecto, Bygren et al. (1996) sugieren que estas prácticas y otras prácticas relacionadas con la actividad cultural provocan una influencia positiva en la supervivencia de las personas. Sin embargo, los resultados del estudio realizado por Linnemann et al. (2017), sugieren que cantar en un coro tiene efectos positivos en el estado de ánimo y el nivel de estrés, pero estos beneficios no tienen por qué estar asociados a las conexiones sociales que se establecen con otros miembros del coro, como han sugerido investigadores anteriores.

El coro es un espacio idóneo para favorecer el crecimiento personal ya que se adquieren habilidades relacionadas con la práctica musical e interpersonal que satisfacen nuestras necesidades de expresión y comunicación. Además, implican un importante sentimiento de responsabilidad ya que no sólo se depende de uno mismo, sino que la pertenencia al grupo nos obliga a pensar en el otro y eso supone un ejercicio de responsabilidad social que puede aplicarse a otras facetas de la vida.

\section{Método}

El objetivo del estudio consiste en realizar un análisis descriptivo de la situación actual de un coro inclusivo universitario, para poder adaptar y diseñar los diferentes tipos de actuaciones que contribuyan a la mejora del bienestar psicológico como compromiso de responsabilidad social.

\subsection{Enfoque metodológico}

El método empleado es identificado de ex post facto, de carácter retrospectivo y comparativo, ya que mediante el mismo se contrastan dos o más grupos según una determinada característica (género, nacionalidad, vinculación, diversidad funcional, etc.) con una variable dependiente (bienestar psicológico).

Antes de realizar el cuestionario, se ofreció a los participantes una breve descripción de la finalidad de la investigación por parte del investigador principal y se facilitó un documento de consentimiento informado 
que garantiza la confidencialidad y el anonimato de los datos recabados en este trabajo, el cual fue leído y firmado por cada uno de los participantes y entregado junto con el cuestionario una vez realizado.

\subsection{Variables}

Las variables objeto de estudio fueron las variables demográficas que son variables categóricas sobre una variable dependiente cuantitativa que es el Bienestar subjetivo de los participantes.

Las variables dependiente sociodemográficas se establecen en función de los datos obtenidos o reportados por el grupo del siguiente modo:

- $\quad$ Género: masculino, femenino, no binario.

- $\quad$ Nacionalidad: Española, Estadounidense (EE.UU.), Rumana, Ecuatoriana, Francesa.

- Vinculación con la Universidad: estudiante de Grado Universitario, Personal Docente e Investigador (PDI), Personal de Administración y Servicios (PAS), Egresado, Estudiante de Máster o posgrado y otra vinculación.

- $\quad$ Presencia de diversidad funcional

- $\quad$ Tipo de diversidad funcional: física, visual, auditiva, intelectual.

Presencia de malestar psicológico

Tipo de malestar psicológico: Trastorno del estado de ánimo (ansiedad o depresión), somatizaciones, Trastorno de Espectro Autista (Síndrome de Asperger), Trastorno del estado de ánimo (ansiedad o depresión) y TDHA, labilidad emocional, trastorno de ánimo y somatizaciones, y otros.

La variable independiente sobre la que se quiere demostrar si existen diferencias significativas es la percepción de bienestar psicológico que, a su vez, está compuesto por los siguientes componentes: autoaceptación, relaciones positivas, autonomía, dominio del entorno, crecimiento personal y propósito en la vida.

\subsection{Muestra y muestreo}

La muestra estuvo compuesta por 73 participantes del coro inclusivo Cantatutti, creado en el año 2017, en la Universidad de Zaragoza, con un enfoque de la práctica coral como medio para el desarrollo personal y de transformación social. Está integrado por personas con y sin diversidad funcional, estudiantes, egresados, profesores, personal de administración y servicios y ciudadanos sin ningún tipo de vinculación con la universidad. Su forma de expresión es multimodal, es decir, mediante la utilización de la voz, el cuerpo y la Lengua de Signos Española (LSE), con el propósito de transmitir su música a un público mayoritario y diverso.

En la actualidad sus componentes tienen edades comprendidas entre los 18 y 68 años, con una edad media de 30,88 años y una desviación típica de 14,75 puntos. El $68,5 \%$ son mujeres y el $28,8 \%$ hombres, lo cual se corresponde con la proporción habitual dentro de una agrupación coral. Un 2,7\% se define como no binario 
(ver Tabla 1). La muestra presenta distinto tipo de vinculación con la Universidad de Zaragoza: estudiantes de Grado (49,4\%), Personal Docente e Investigador (PDI) (8,2\%), Personal de Administración y Servicios (PAS) $(5,5 \%)$, egresado $(23,3 \%)$, estudiantes de posgrado o máster $(6,8 \%)$ y otro tipo de vinculación $(6,8 \%)$. En cuanto al origen de los participantes, la mayoría $(94,5 \%)$ son españoles. El porcentaje restante son de EEUU $(1,4 \%)$, Ecuador $(1,4 \%)$, Rumania $(1,4 \%)$ y Francia $(1,4 \%)$.

La muestra está conformada por personas con y sin diversidad funcional, existe un $86,6 \%$ sin discapacidad y un $13,7 \%$ con algún tipo de discapacidad: visual (4.4\%), auditiva $(1,4 \%)$, psíquica $(2,7 \%)$ y física $(4,1 \%)$.

En relación con la presencia de malestar psicológico indicado por los participantes, se han obtenido los siguientes datos: ningún malestar (74\%), Trastorno del estado de ánimo (ansiedad o depresión) (5,5\%), dificultades a nivel somático (2,7\%), Trastorno del Espectro Autista (1,4\%), Trastorno del estado de Ánimo y TDHA (2,7\%), labilidad emocional (5,5\%), Trastorno del estado de ánimo y somatizaciones (2,7\%).

Los datos se obtuvieron en un periodo de una semana. El pase de pruebas se realizó por un investigador de la Universidad de Zaragoza (UZ) y el tipo de muestreo fue intencional.

Tabla 1 - Valores de edad y género del coro Cantatutti

\begin{tabular}{|lllll|}
\hline & Mínimo & Máximo & Media & Desv. \\
\hline Edad & 18 & 68 & 30,88 & 14,75 \\
\hline & Género & Frecuencia & Porcentaje & Porcent Acum \\
\hline Género & Mujer & 50 & 68,5 & 68,5 \\
\hline & Hombre & 21 & 28,8 & 97,3 \\
\hline & No binario & 2 & 2,7 & 100 \\
\hline & Total & 73 & 100 & \\
\hline
\end{tabular}

\subsection{Instrumentos}

La recogida de datos se realizó a través de la adaptación española de la Escala de bienestar psicológico de Ryff (Díaz et al. 2006). La escala está compuesta por 39 ítems con opciones de respuesta tipo Likert de 6 puntos ( $1=$ totalmente en desacuerdo y $6=$ totalmente de acuerdo), incluyendo seis sub-escalas: Autoaceptación (AUTOAC) (6 ítems); Relaciones positivas (RELS) (6 ítems); Autonomía (AUT) (8 ítems); Dominio del entorno (EN) (6 ítems); Crecimiento personal (CREC) (7 ítems) y; Propósito en la vida (PROP) (6 ítems). La consistencia interna de las escalas como muestra el alfa de Cronbach es de: 0,83 (autoaceptación), 0,88 (relaciones positivas), 0,87 (autonomía), 0,90 (dominio del entorno), 0,68 (crecimiento personal) y 0,80 (propósito en la vida).

\section{Resultados}

Según el objetivo principal de este trabajo, se ha realizado un análisis descriptivo del bienestar psicológico y, en segundo lugar, se ha realizado un Análisis de Varianza para averiguar la posible existencia de diferencias significativas en el bienestar psicológico en las distintas variables sociodemográficas establecidas (vinculación con la universidad, género, nacionalidad, presencia de discapacidad y tipo de discapacidad, malestar psicológico y tipo de malestar y género). Para el análisis estadístico se utilizó el programa SSPS 26. 
Los estadísticos utilizados han sido pruebas descriptivas y el análisis de varianza de Fisher (distribución F de Fisher como parte del contraste de hipótesis).

En la Tabla 2 se muestran los valores de los estadísticos descriptivos obtenidos del bienestar psicológico de los participantes (media, desviación típica, valor máximo y mínimo). Según se puede ver, el valor medio del bienestar psicológico del grupo en su totalidad es de 176,58 puntos. En función de la variable que se analice se va a observar una puntuación por encima o debajo de dicho valor, esto nos da una muestra del grado de bienestar psicológico con respecto a ella.

Tabla 2 - Valores descriptivos del bienestar psicológico en las variables sociodemográficas

\begin{tabular}{|c|c|c|c|c|c|c|c|c|c|c|}
\hline Valor & Grupo & Mujeres & Varones & $\begin{array}{c}\text { No } \\
\text { binario }\end{array}$ & España & EEUU & Ecuador & Rumanía & Francia & \\
\hline Media & 176,58 & 180,4 & 169,57 & 154,5 & 178,20 & 146 & 130 & 159 & 159 & \\
\hline Desv. & 21,03 & 18,66 & 24,38 & 12,02 & 20,27 & & & & & \\
\hline Mín-Máx. & $126-212$ & $133-212$ & $126-201$ & $146-263$ & $126-212$ & & & & & \\
\hline Vinculación & Grado & PDI & PAS & Egresado & Máster & Otros & & & & \\
\hline Media & 178,42 & 190,67 & 170,75 & 171,65 & 176,40 & 173,20 & & & & \\
\hline Desv. & 19,96 & 15,00 & 28,96 & 21,05 & 10,21 & 27,44 & & & & \\
\hline Mín-Máx. & 134-209 & $171-203$ & $133-200$ & $139-212$ & 163-186 & $\begin{array}{l}126- \\
201\end{array}$ & & & & \\
\hline $\begin{array}{l}\text { Diversidad } \\
\text { Funcional }\end{array}$ & $\begin{array}{c}\text { SIN } \\
\text { DISC }\end{array}$ & DISC & Visual & Auditiva & Psíquica & Física & & & & \\
\hline Media & 177,38 & 171,50 & 157,00 & 197,00 & 164,50 & 187,00 & & & & \\
\hline Desv. & 19,90 & 27,85 & 34,71 & & 10,61 & 21,70 & & & & \\
\hline Mín-Máx. & $133-212$ & $126-201$ & 126-197 & & $157-172$ & $\begin{array}{l}162- \\
201\end{array}$ & & & & \\
\hline $\begin{array}{l}\text { Malestar } \\
\text { psicológico }\end{array}$ & $\begin{array}{c}\text { No } \\
\text { acuse } \\
\text { malestar }\end{array}$ & $\begin{array}{l}\text { Acuse } \\
\text { malestar }\end{array}$ & Ánimo & Somático & TEA & $\begin{array}{c}\text { Ánimo } \\
\text { y } \\
\text { TDHA }\end{array}$ & Labilidad & $\begin{array}{l}\text { Ánimo y } \\
\text { somat }\end{array}$ & Bipolar & Otros \\
\hline Media & 181,70 & 162,00 & 162,50 & 146,00 & 172,00 & 143,50 & 158,25 & 147,50 & 174,00 & 191,67 \\
\hline Desv. & 17,65 & 23,41 & 14,48 & 18,38 & & 6,36 & 35,44 & 7,78 & & 15,37 \\
\hline Mín-Máx. & $130-212$ & $126-203$ & $146-180$ & $133-159$ & & $\begin{array}{c}139- \\
148\end{array}$ & $126-203$ & $142-153$ & & $\begin{array}{l}174- \\
202\end{array}$ \\
\hline
\end{tabular}

En los gráficos, se puede visualizar el grado de bienestar psicológico según las distintas variables analizadas: género, nacionalidad, vinculación con la universidad, diversidad funcional y malestar psicológico.

En la Figura 1, se puede ver que el género no binario es el que más baja puntuación ha obtenido de bienestar psicológico (Media=154,5; SD=12,02), seguido de los varones (Media=169,57) y finalmente, de las mujeres (Media $=180,4 ; S D=18,66$ ). 


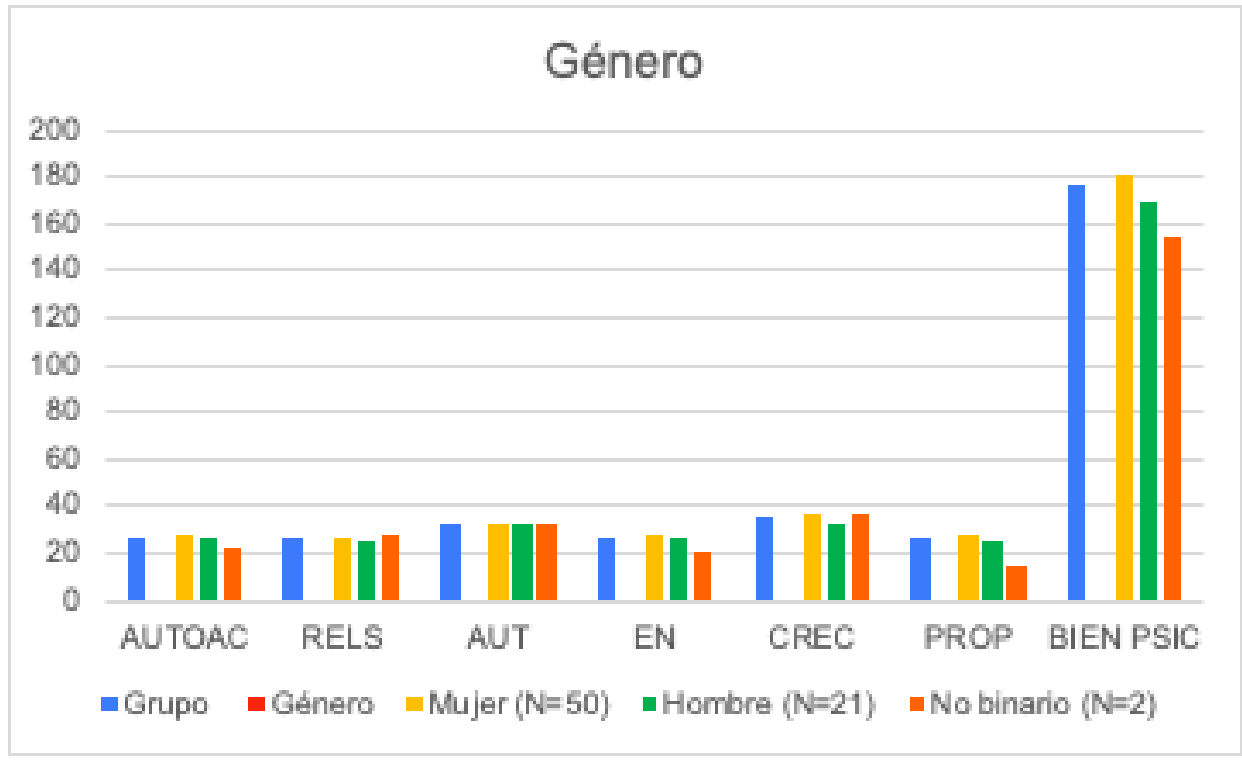

Figura 1 - Bienestar psicológico en función del género

En relación a la vinculación con la universidad (Figura 2), no se pueden apreciar grandes diferencias, siendo el Personal Docente e Investigador el que presenta mayores niveles de bienestar psicológico percibido con respecto al resto de grupos vinculados (Media=190,67; SD=15).

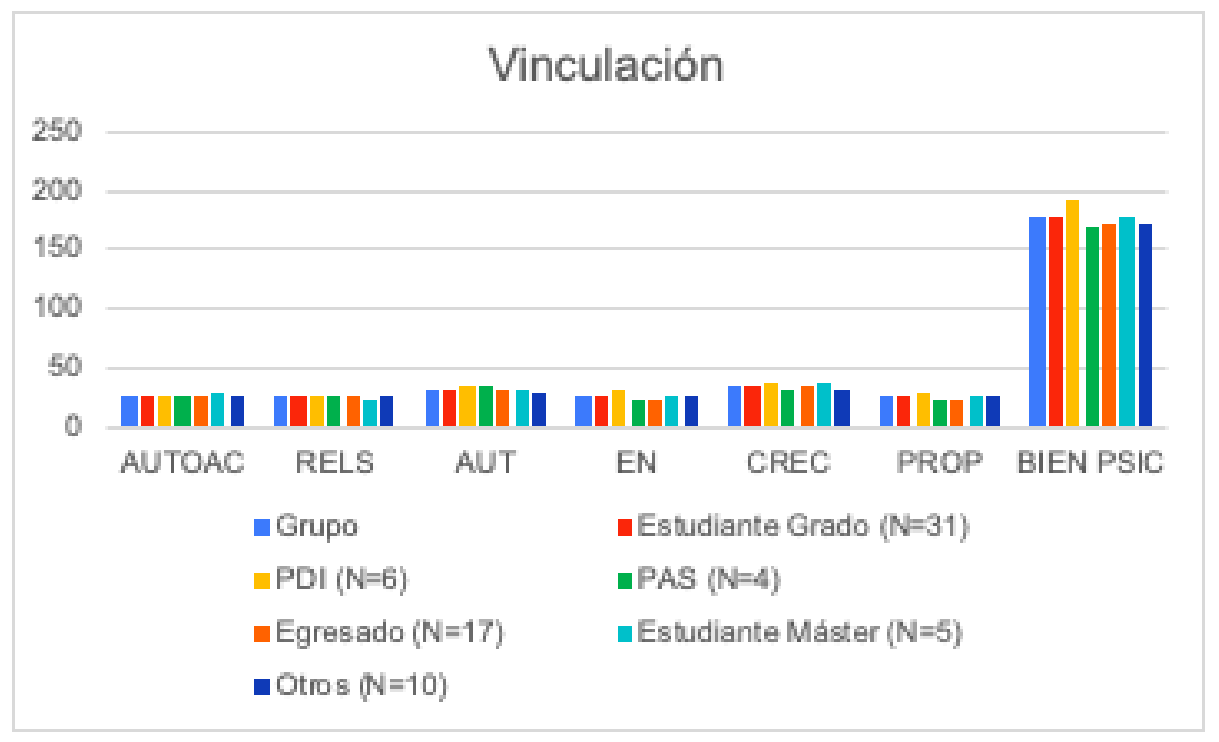

Figura 2 - Bienestar psicológico en función de la vinculación con la UZ

Según las personas con diversidad funcional (Figura 3) se puede observar que las personas con algún tipo de discapacidad, presentan un bienestar psicológico percibido menor (Media=171,50; SD=27,85) en relación a las personas sin diversidad funcional (Media=177,98; SD=19,90). 


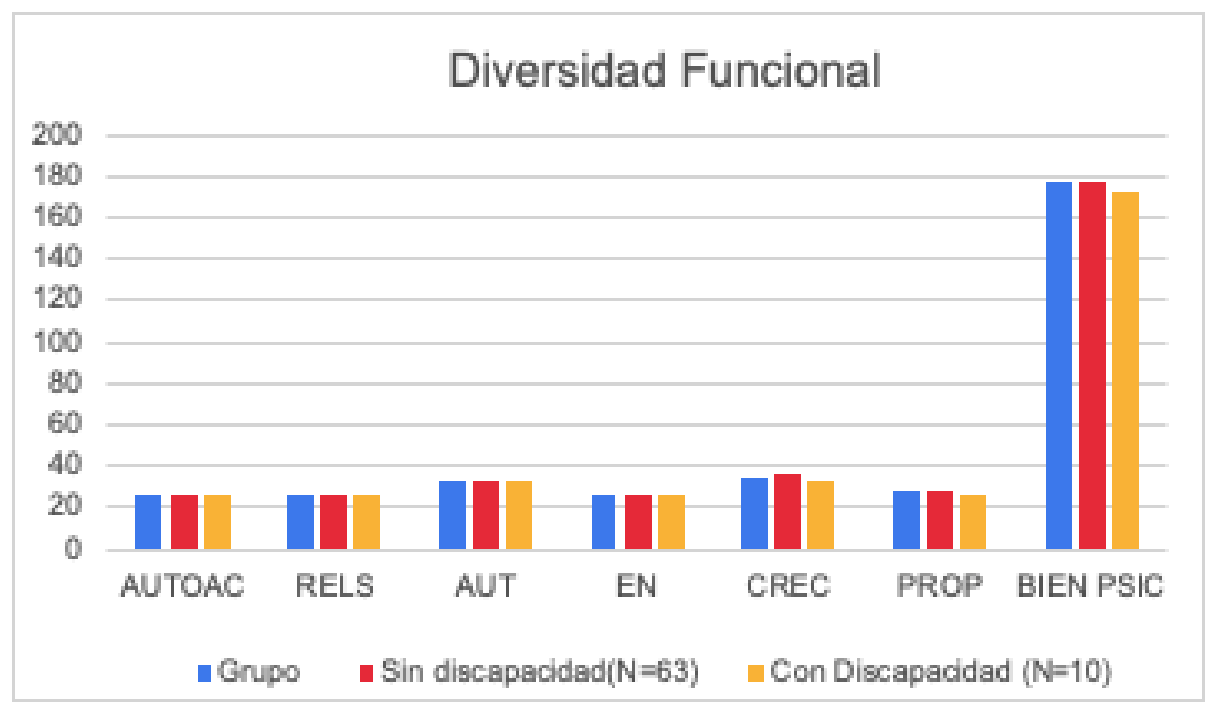

Figura 3 - Bienestar psicológico en las personas con diversidad funcional

Con respecto a las personas que no acusan algún tipo de malestar a nivel psíquico (Figura 4), el bienestar psicológico percibido es mayor (Media= 181,70; SD=17,65) que el de las personas que padecen algún tipo de malestar (Media= 162; SD= 23,41).

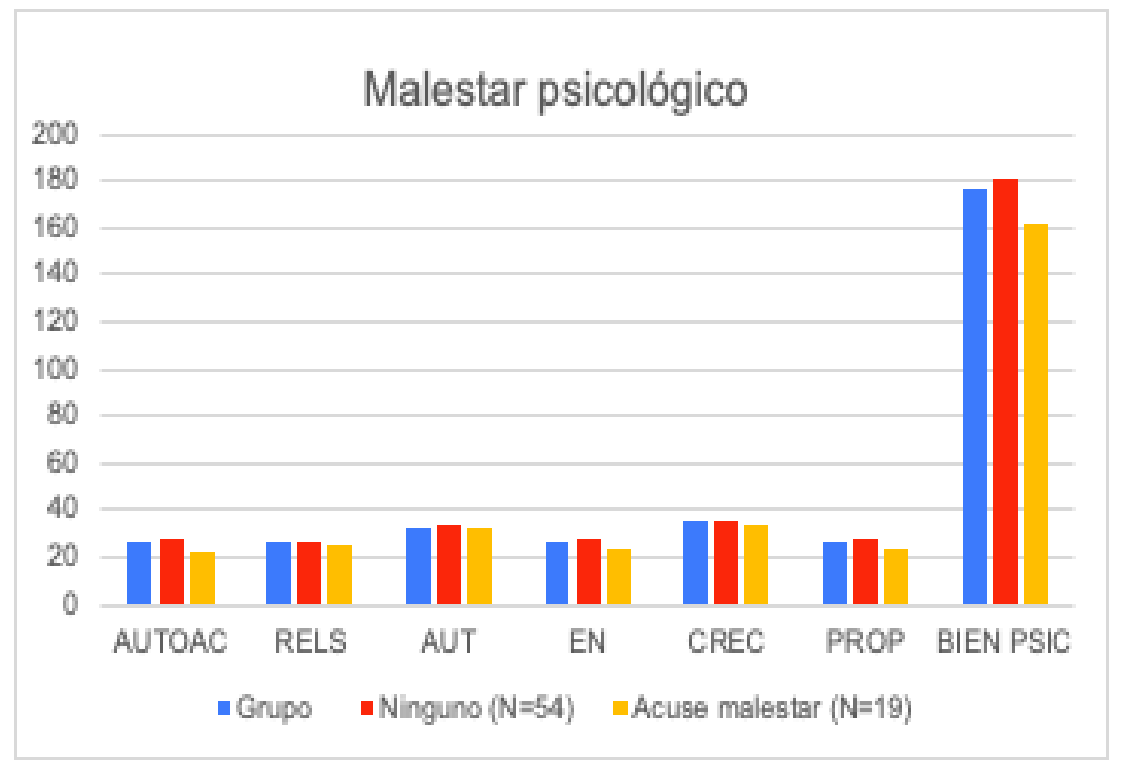

Figura 4 - Bienestar psicológico en las personas que acusan malestar

Asimismo, las personas con menor bienestar psicológico son aquellas personas que presentan algún trastorno de ánimo con TDHA (Media=143,50; SD=6,36) o algún tipo de trastorno somático (Media=146; $S D=18,38$ ) o algún trastorno de ánimo y somatizaciones (Media= 147,59; SD=7,78) (Figura 5). 


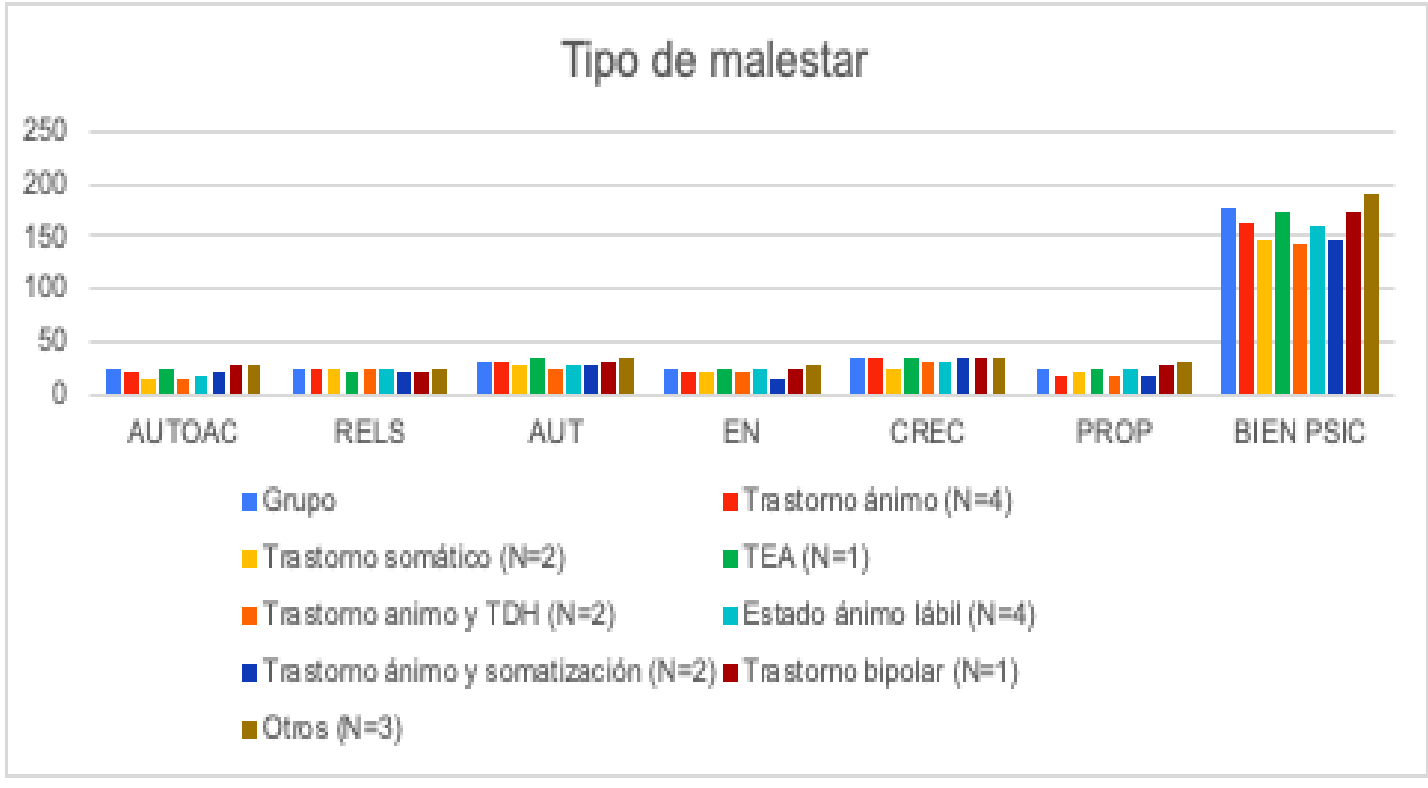

Figura 5 - Bienestar psicológico en función del tipo de malestar psíquico

Entre los distintos grupos de estudio (ver Tabla 3), no se han hallado diferencias significativas en el bienestar psicológico percibido según el tipo de vinculación con la universidad ( $F=0,9 ;$ Sig.: 0,5), así como en ninguno de sus componentes: Autoaceptación ( $F=0,57$; Sig. $=0,72)$; Relaciones positivas $(F=0,42$; Sig. $=0,83$ ); Autonomía ( $F=0,94$; Sig.= 0,46); Dominio del Entorno ( $F=1,57$; Sig.=0,18); Crecimiento $(F=1,42$; Sig.=0,23) y; Propósito en la vida ( $F=1.04 ;$ Sig.= 0,4).

En el resto de variables analizadas, se han hallado diferencias significativas en algunos de los componentes del bienestar psicológico y en otros, en algunos de sus componentes, así como en el bienestar psicológico percibido.

En relación al género, se han observado diferencias significativas entre hombres y mujeres y no binarias en el crecimiento psicológico $(F=4,23$; Sig. $=0,02)$, propósito en la vida $(F=6,4$; Sig.= 0,003) y en el bienestar psicológico de forma global $(\mathrm{F}=3,3 ; \mathrm{Sig} .=0,04)$. Como apuntábamos anteriormente, las mujeres son las que mayor bienestar psicológico se ha encontrado y estas diferencias frente a los varones y personas no binarias son significativas.

En relación a la nacionalidad, las diferencias encontradas también son significativas. Entre ellas, la puntuación más elevada se encuentra en la población de nacionalidad española $(M=178,20 ; S D=20,27)$ con diferencias significativas en las variables de Dominio del entorno $(F=2,8$; Sig. $=0,03)$, Crecimiento personal $(F=2,6$; Sig.= 0,01) y Propósito en la vida ( $F=3,3 ;$ Sig.= 0,01).

En relación a las personas con y sin diversidad funcional, no hay diferencias significativas en el bienestar psicológico percibido $(F=0,67$; Sig. $=0,41)$. En la única variable en la que se han hallado diferencias significativas, es en la de crecimiento personal ( $F=5,99$; Sig. $=0,017)$.

Es importante destacar las diferencias percibidas en el bienestar psicológico en relación al malestar psicológico indicado. Se han hallado diferencias significativas en el bienestar psicológico percibido de las personas que acusan algún tipo de malestar con respecto a las personas que no acusan algún tipo de 
malestar a nivel psicológico $(F=14,69 ;$ Sig. $=0,000)$, así como en los componentes de Autoaceptación $(F=26,17$; Sig.= 0.000), Relaciones positivas ( $F=4,38$; Sig.=0,040), Dominio del entorno $(F=13,94$; Sig. $=0,000)$ y Propósito en la vida $(F=7,63$; Sig.=0,010). El componente en el que no se han hallado diferencias significativas es en el de crecimiento personal $(F=0,80$; Sig. $=0,380)$.

Tabla 3. Diferencias entre bienestar psicológico y valores sociodemográficos, diversidad funcional y malestar psicológico.

\begin{tabular}{|c|c|c|c|c|c|c|c|}
\hline Género & AUTOAC & RELS & AUT & EN & CREC & PROP & $\begin{array}{l}\text { BIEN } \\
\text { PSIC }\end{array}$ \\
\hline $\mathbf{F}$ & 1,63 & 2,85 & 0,02 & 2,87 & 4,23 & 6,4 & 3,3 \\
\hline Sig & 0,2 & 0,06 & 0,98 & 0,06 & $\mathbf{0 , 0 2}$ & 0,003 & 0,04 \\
\hline Nacionalidad & AUTOAC & RELS & AUT & EN & CRE & PROP & BIEN PSIC \\
\hline $\mathbf{F}$ & 2,3 & 0,97 & 1,07 & 2,8 & 2,6 & 3,3 & 2,4 \\
\hline Sig. & 0,07 & 0,43 & 0,4 & 0,03 & 0,04 & $\mathbf{0 , 0 1}$ & 0,06 \\
\hline Vinculación & AUTOAC & RELS & AUT & EN & CREC & PROP & BIEN PSIC \\
\hline $\mathbf{F}$ & 0,56 & 0,42 & 0,94 & 1,58 & 1,42 & 1,04 & 0,90 \\
\hline Sig. & 0,72 & 0,83 & 0,46 & 0,18 & 0,23 & 0,40 & 0,50 \\
\hline $\begin{array}{l}\text { Diversidad } \\
\text { Funcional }\end{array}$ & AUTOAC & RELS & AUT & EN & CREC & PROP & BIEN PSIC \\
\hline $\mathbf{F}$ & 0,02 & 1,30 & 0,09 & 0,00 & 5,99 & 0,01 & 0,67 \\
\hline Sig & 0,887 & 0,260 & 0,762 & 0,948 & $\mathbf{0 , 0 1 7}$ & 0,912 & 0,41 \\
\hline $\begin{array}{l}\text { Malestar } \\
\text { psicológico }\end{array}$ & AUTOAC & RELS & AUT & $\mathrm{EN}$ & CREC & PROP & BIEN PSIC \\
\hline $\mathbf{F}$ & 26,17 & 4,38 & 1,69 & 13,94 & 0,80 & 7,63 & 14,69 \\
\hline Sig & $\mathbf{0 , 0 0 0}$ & 0,040 & 0,200 & 0,000 & 0,380 & $\mathbf{0 , 0 1 0}$ & 0,000 \\
\hline
\end{tabular}

\section{Discusión y Conclusiones}

Según los resultados obtenidos a través de la adaptación de la escala Ryff de Díaz et al. (2006) sobre el bienestar psicológico, podemos extraer diferentes conclusiones relacionadas con el objetivo de estudio.

En primer lugar, atendiendo a las variables que no muestran diferencias significativas, encontramos la vinculación con la universidad ( $F=0,9$; Sig.: 0,5$)$ y la diversidad funcional $(F=0,67 ;$ Sig. $=0,41)$. Por lo tanto, ni la vinculación con la universidad ni la diversidad funcional parecen ser aspectos que impliquen un mayor o menor bienestar psicológico per se. Inicialmente, las personas con diversidad funcional, no son aquellas que perciben menor bienestar psicológico de forma significativa con respecto a aquellas sin diversidad funcional, a pesar de que sus niveles de bienestar psicológico percibido sean menores (Media=171,50; SD=27,85) en comparación con las personas sin diversidad funcional (Media=177,98; SD=19,90) (figura 3). Estos datos nos permiten formular la hipótesis de que la actividad desarrollada en el coro inclusivo Cantatutti podría favorecer la equiparación en cuanto al bienestar psicológico de sus miembros en las variables de vinculación con la universidad y diversidad funcional. Esta cuestión es interesante en relación a las actividades que se plantean en el coro como propuesta de cambio social, ya que se podría favorecer la equiparación de los valores de bienestar psicológico en aras de un mayor bienestar social, por tanto, de una mayor inclusión de sus integrantes en favor de la justicia social. Tal y como apunta Uribe (2004), los dos conceptos de bienestar desde el nivel individual al colectivo, están íntimamente entrelazados, por lo que se podría proponer un itinerario de confluencia entre ellos enmarcado en los derechos ciudadanos y del contrato social. 
En segundo lugar, es importante señalar que sí se aprecian diferencias significativas en el bienestar psicológico con respecto a la diversidad de género y la presencia de malestar psicológico. Si nos centramos en el género, las mujeres han mostrado un mayor bienestar psicológico con respecto a los hombres y el género no binario. Tal y como se ha podido mostrar en la figura 1, el género no binario es el que más baja puntuación ha obtenido en su bienestar psicológico (Media=154,5; SD=12,02), seguido de los varones (Media= 169,57 y SD: 24,38) y finalmente, de las mujeres (Media=180,4; SD=18,66), que son las que presentan mayor bienestar psicológico, además dichas diferencias son estadísticamente significativas ( $F=3,3$; $\mathrm{F}=0,04)$ así como en los componentes de crecimiento psicológico $(\mathrm{F}=4,23 ;$ Sig. $=0,02)$ y propósito en la vida $(F=6,4 ;$ Sig. $=0,003)$. Estos resultados están en consonancia con los estudios realizados por Clift et al. (2010b) y Pérez-Aldeguer (2014), en los que las mujeres mostraron un mayor bienestar psicológico que los hombres en todas las variables analizadas. Si estos datos son extrapolables a otros coros, estas diferencias se podrían relacionar con la presencia de mayor número de componentes femeninos que varones en las agrupaciones corales.

Por último, se han registrado diferencias significativas en el bienestar psicológico percibido de las personas que presentan algún tipo de malestar psicológico con respecto a aquellas personas que no lo presentan ( $F=14,69$; Sig. 0,000$)$, así como en los componentes de autoaceptación ( $F=26,17$; Sig. $=0.000)$; relaciones positivas $(F=4,38$; Sig. $=0,040)$, dominio del entorno $(F=13,94$; Sig. $=0,000)$ y propósito en la vida $(F=7,63$; Sig. $=0,010)$ exceptuando el de crecimiento personal $(F=0,80 ;$ Sig. $=0,380)$.

Algunos estudios identifican como variables predictoras de un elevado bienestar psicológico, aspectos relacionados con la personalidad tales como un buen autoconcepto y autoestima, bienestar material, extraversión y bajo neuroticismo. Por lo que las intervenciones que fomenten el autoconcepto, la autoestima y desarrollo identitario así como la extraversión, contribuyen al bienestar y satisfacción personal (Garaigordobil et al. 2009; Oriola-Requena, Calderón-Garrido, Gustems-Carnicer 2021). En otros estudios se han proporcionado evidencias de los beneficios de un mayor bienestar afectivo de las personas que han practicado música como hobbie (Koehler \& Neubauer 2020). Estos resultados sugieren que la satisfacción de las necesidades psicológicas básicas son un mecanismo mediador y enfatizan la importancia de distinguir entre los indicadores de afecto positivo y satisfacción y de afecto negativo e insatisfacción.

A su vez, se insiste en la relación entre bienestar psicológico y social promoviendo una mayor justicia social, aspecto esencial de este trabajo (Cooper 2020; Murillo y Hernández-Castilla 2014; Uribe 2004). Se promueve, por tanto, una visión acorde a los beneficios emocionales procurados por la actividad coral, contrastada en diversos estudios (Bygren, Konlaan \& Johansson 1996; Hallam 2010; Linnemann, Schnersch y Nater 2017) y, por tanto, estas diferencias podrían tender a desaparecer en un futuro debido a la propia actividad coral.

Como limitaciones a este estudio se desprende que se ha realizado solamente una toma de datos, sin prepost por dos motivos. El primero, la variabilidad de la muestra que hace imposible comparar a los participantes consigo mismos de forma suficientemente espaciada en el tiempo. Y, por otro lado, porque el objetivo de la toma de datos es implementar medidas o modificaciones en las acciones que puedan llevar a una mejora de los indicadores medidos sobre bienestar subjetivo.

Podemos concluir que este estudio ha cumplido con el objetivo planteado inicialmente de realizar un análisis descriptivo de la situación actual del coro inclusivo Cantatutti de la Universidad de Zaragoza. Sirve esto como 
punto de partida para la implementación activa de nuevas actividades y metodologías que ayuden a la mejora del bienestar de sus miembros, intentando equilibrar las diferencias significativas observadas en cuanto al género o los diversos malestares indicados por sus participantes. Todo ello con la finalidad de alcanzar una mayor justicia social entre la población activa del proyecto.

Este estudio puede ser el primer paso o recogida de datos inicial para posteriores mediciones e investigaciones que comparen el mismo coro en distintos momentos, o al coro con otros ciudadanos que no participen de actividades musicales o cualquier tipo de actividad grupal. Debemos comprometernos con la sociedad en la creación de agrupaciones musicales que promuevan la participación y la creación de ambientes saludables, que sean sensibles a la percepción positiva de las diferencias y a las necesidades sociales de cada comunidad, es decir, con una visión caleidoscópica de la justicia y la responsabilidad social.

\section{Referencias}

Blanco, Amalio y Darío Díaz. 2006. “El bienestar social: su concepto y medición". Psicothema 17(4): 582-589. www.psicothema.com

Broh, Beckett A. 2002. "Linking extracurricular programming to academic achievement: who benefits and why?" Sociology of Education 75: 69-95. https://doi.org/10.2307/3090254

Bygren, Lars O., Boinkum B., Konlaan \& Sven-Erik Johansson 1996. "Attendance at cultural events, reading books or periodicals, and making music or singing in a choir as determinants for survival: Swedish interview survey of living conditions". BMJ Clinical Research 313:7072. https://doi.org/10.1136/bmj.313.7072.1577

Cámara, Aintzane. 2003. "El canto colectivo en la escuela: una vía para la socialización y el bienestar personal". Revista psicodidáctica, 15-16: 105-110. https://www.redalyc.org/pdf/175/17515081007.pdf

Clift, Stephen M. and Grenville Hancox. 2001. "The perceived benefits of singing: findings from preliminary surveys of a university college choral society". Journal of the Royal Society for the Promotion of Health 121 (4): 248-256. https://doi.org/10.1177/146642400112100409

Clift, Stephen, Jennifer Nicols, M. Raisbeck, Christine Whitmore and lan Morrison. 2010a. "Group singing, wellbeing and health: Asystematic review". The UNESCO Journal 2: 1-25.

Clift, Stephen, Grenville Hancox, lan Morrison, Brbel Hess, Gunter Kreutz and Don Stewart. 2010b. “Choral singing and psychological wellbeing: Quantitative and qualitative findings from English choirs in a crossnational survey". Journal of Applied Arts and Health 1 (1): 19-34. https://doi.org/10.1386/jaah.1.1.19/1

Cohen, Gene D., Susan Perlstein, Jeff Chapline, Jeanne Kelly, Kimberly M. Firth, and Samuel Simmens. 2006. "The impact of professionally conducted cultural programs on the physical health, mental health, and social functioning of older adults". The Gerontologist 46: 726-734. https://doi.org/ 10.1093/geront/46.6.726

Cooper, Mick. 2020. "Directionality: Unifying Psychological and Social Understandings of Well-Being and Distress Through an Existential Ontology". Journal of Humanistic Counseling 60: 6-25. https://doi.org/10.1002/johc.12148

Díaz, Darío, Raquel Rodríguez-Carvajal, Amalio Blanco, Bernardo Moreno-Jiménez, Ismael Gallardo, Carmen Vall y Dirk van Dierendonck. 2006. “Adaptación española de las escalas de bienestar psicológico de Ryff". Psicothema, 18: 572-577. 
Díaz, Juan y Sánchez Mạ Pilar. 2002. "Relaciones entre estilos de personalidad y satisfacción autopercibida en diferentes áreas vitales". Psicothema, 14: 100-105.

Diener, Edward. 1994. “El bienestar subjetivo”. Intervención psicosocial, 3: 67-113.

Dingle, Genevieve, Stephen Clift, Saoirse Finn, Rebakah Gilbert, Jenny Groarke, Elyse J. Williams, et al. 2019. "An Agenda for Best Practice Research on Group Singing, Health, and Well-Being". Music \& Science 2: 1-10. https://doi.org/10.1177/2059204319861719

Elefant, Cochavit, Meir Lotan, Felicity A. Baker and Geir Olve Skeie. 2012. "Effects of music therapy on facial expression of individuals with Parkinson's disease: A pilot study". Musicae Scientiae 16(3): 392-400. https://doi.org/10.1177/1029864912458917

Eyre, Lillian. 2011. "Therapeutic Chorale for Persons with Chronic Mental Illness: A Descriptive Survey of Participant Experiences". Journal of Music Therapy 48(2): 149-168. https://doi.org/10.1093/jmt/48.2.149

Fernández-Herranz, Nuria S. 2013. Las agrupaciones corales y su contribución al bienestar de las personas: percepción de las aportaciones del canto coral a través de una muestra de cantores. [Tesis no publicada, Universidad Carlos III]. Repositorio institucional http://hdl.handle.net/10016/18185

Garaigordobil, Maite, Jone Aliri e Iraide Fontaneda. 2009. "Bienestar psicológico subjetivo: diferencias de sexo, relaciones con dimensiones de personalidad y variables predictoras". Psicología Conductual 17(3): 543-559.

Gelabert Gual, LLorenc. 2017. "La práctica del canto colectivo como eje transversal de conocimientos, actitudes y valores: una propuesta dirigida a alumnos de Grado en Educación Infantil y Primaria". Foro de Educación 15, (22): 1-21. http://dx.doi.org/10.14516/fde.505

Hallam, Susan. 2010. "The power of music: Its impact on the intellectual, social and personal development of children and young people". International Journal of Music Education 28(3): 269-289. https://doi.org/10.1177/0255761410370658

Juan-Morera, Borja, Icíar, Nadal-García y M. Belén López-Casanova. 2020. "Música y lengua de signos a cuatro voces, una experiencia educativa y musical para la inclusion". Revista Electrónica de LEEME 45: 35-52. https://doi.org/10.7203/LEEME.45.16244

Koehler, Friederike \& Andreas B., Neubauer. 2020. "From music making to affective well-being in everyday life: The mediating role of need satisfaction". Psychology of Aesthetics, Creativity, and the Arts 14(4): 493-505. https://doi.org/10.1037/aca0000261

Kuhn, Dawn. 2002. "The effects of active and passive participation in musical activity on the immune system as measured by salivary immunoglobulin A(SigA)". Journal of Music Therapy 39 (1): 3039.https://doi.org/10.1093 / jmt / 39.1.30

Lacárcel, Josefa. 2003. "Psicología de la música y emoción musical". Educatio Siglo XXI 20: 213-226. https://revistas.um.es/educatio/article/view/138

Lally, Elaine. 2009. "The power to heal us with a smile and a song': Senior well-being, music-based participatory arts and the value of qualitative evidence". Journal of Arts and Communities 1 (1): 25-44. https://doi: 10.1386/jaac.1.1.25/1

Linnemann, Alexandra, Anna, Schnersch \& Urs Nater M. 2017. "Testing the beneficial effects of singing in a choir on mood and stress in a longitudinal study: The role of social contacts". Musicae Scientiae 21(2): 213-229. https://doi.org/10.1177/1029864917693295 
Lonslade, Adam J. \& Evelyn R. Day. 2020. "Are the psychological benefits of choral singing unique to choirs? A comparison of six activity groups". Psychology of Music 1-20 https://doi.org/10.1177/0305735620940019

Muhip \& Gorseu 2014. "Psychological and physiological effects of singing in a choir". Psychology of Music 42: 420-429.https://doi.org/10.1177/0305735613477181

Mujica, Felipe, Claudia, Inostroza, C y Nelly Orellana. 2018. "Educar las emociones con un sentido pedagógico: un aporte a la justicia social". Revista Internacional de Educación para la Justicia Social 7(2): 113-127. https://doi.org/10.15366/riejs2018.7.2.007

Murillo, Francisco Javier y Reyes Hernández-Castilla. 2014. "Liderando escuelas justas para la justicia social”. Revista Internacional de Educación para la Justicia Social 3(4): 13-32. https://revistas.uam.es/riejs/article/view/33

Oriola-Requena, Salvador; Diego, Calderón-Garrido, Diego; Josep, Gustems-Carnicer. 2021. "Claves para la participación de los adolescentes en agrupaciones musicales juveniles: el caso de los coros en Catalunya y las bandas en Valencia" Per Musi 41:1-15. https://doi.org/10.35699/2317-6377.2021.29206

Pearce, Eiluned, Jacques, Launay, Anna, Machin y Robin I.M. Dunbar. 2016. "Is group singing special? Health, well-being and social bonds in community-based adult education classes". Journal of Community \& Applied Social Psychology 26: 518-533. https://doi.org/10.1002/casp.2278.

Pérez-Aldeguer, Santiago. 2014. "Los efectos del canto coral sobre el bienestar psicológico en adultos mayores". Revista argentina de clínica psicológica 23 (3): 199-208.

Pieridou, Skoutella. 2018. A multicultural/intercultural conceptual framework for training music professionals for disadvantage youth, social inclusion and music - Suggested activities and model of implementation Erasmus+ KA2 STAMP Project - Social Inclusion in Music. https://stamp-music.org/wpcontent/uploads/2018/12/Avra-\%C3\%ADr\%C3\%A1sa.pdf

Ryff, Carol. 1989a. "Beyond Ponce de Leon and life satisfaction: New directions in quest of successful aging". International Journal of Behavioral Development 12: 35-55. https://doi.org/10.1177/016502548901200102

Ryff, Carol. D. 1989b. "Happiness is everything, or is it? Explorations on the meaning of psychological wellbeing". Journal of Personality and Social Psychology 57: 1069-1081. https://doi.org/10.1037/00223514.57.6.1069

Sarfson, Susana y Natalia Larraz. 2017. "Influencias del aprendizaje musical en el bienestar de un grupo de personas mayores de 65 años". Acta de investigación psicológica 7:2727-2734 http://www.psicologia.unam.mx/acta-de-investigacion-psicologica/

Uribe, Consuelo. 2004. "Desarrollo social y bienestar". Universitas Humanística 31(58): 1125.https://revistas.javeriana.edu.co/index.php/univhumanistica/article/view/9509

Valentine, Elizabeth and Claire Evans. 2001. "The effects of solo singing, choral singing and swimming on mood and physiological indices". British Journal of Medical Psychological Society 74 (1): 115-120. https://doi.org/10.1348/000711201160849

Varvarigou, Maria. 2018. Group playing by ear from recordings as a vehicle for the social inclusion of disadvantaged youth. Erasmus + KA2 STAMP Project, 1-12. https://stamp-music.org/wpcontent/uploads/2018/12/Maria-Varvarigou-\%C3\%ADr\%C3\%A1sa.pdf

Whitwell, David. 1977. Music learning through performance. Texas: Texas Music Educators Association. 